\title{
PLATELETS ACTIVATION IN PATIENTS UNDERGOING PTCA AND THEIR RESPONSIVENESS AFTER IN VITRO STIMULATION
}

\author{
Martina Loudová, Jan Krejsek \\ Charles University in Prague, Faculty of Medicine in Hradec Králové: Department of Clinical Immunology and Allergology
}

\begin{abstract}
Summary: We evaluated expression of platelet activation markers in blood samples of 15 patients who underwent percutaneous transluminal coronary angioplasty (PTCA) by flow cytometry. Analysis was performed before the beginning of PTCA, during initial coronary angiography and after the end of PTCA or after a stent placement, respectively. We evaluated platelet-derived microparticles, platelet-leukocyte aggregates, platelet aggregates and a membrane expression of CD62P and CD63 molecules. Responsiveness of platelets to the activation in vitro with thrombin-receptor activating protein - 6 (TRAP-6) was tested simultaneously. Statistically significant differences between patient samples were found only in the expression of the activation markers CD62P (before PTCA $0.22 \%$, during $0.39 \%$, after $0.67 \%$ ), CD63 (0.26 \%/ $0.45 \% / 0.85 \%$ ) and platelet-leukocyte aggregates ( $13.57 \% / 18.39 \% / 23.63 \%$ ).In the same group the expression of all constitutive membrane markers was statistically significantly decreased: in patients undergoing PTCA was the expression of CD9:87.98 \% (in comparison with control group 94.98 \%), CD31: 87.10 \% (92.78 \%), CD36: 87.37 \% (90.98 \%), CD41: 88.09 \% (95.62 \%), CD42a: 88.54 \% (94.98 \%), CD42a: $88.31 \%$ (94.13\%).
\end{abstract}

Key words: Platelets; Activation markers; Angioplasty; Flow cytometry; Platelet activation in vitro

\section{Introduction}

PTCA is an invasive procedure during that dilated balloon is causing a „controlled lesion“ in the arterial wall narrowed by stenosis. Endothelial cell-lining of artery is damaged by inflated balloon and is changed from thromboresistant to prothrombogenic. Thrombi could be formed which are, in the most aggravated situation, responsible for the occlusion of dilated artery $(6,18,29)$. The activation of platelets can be followed by flow cytometry. The formation of heteroaggregates between platelets and leukocytes, platelets homoaggregates together with release of procoagulant platelet microparticles could be determined. The ample evidence of previous platelet activation is simultaneous membrane expression of both P-selectin (CD62P) adhesion molecule and CD63 receptor on the surface of platelets $(1,9,24,26,27)$. In patients who underwent PTCA we followed before procedure, during and after finishing of PTCA, the expression of the constitutively expressed platelet membrane molecules CD9, CD31, CD36, CD41, CD42a and CD42b, and activationinduced platelet membrane molecules CD62P and CD63. Other markers of platelet activation such as platelet microparticles (MPs), platelet homoaggregates and aggregates between platelets and leukocytes (PLAs) were also determined by flow cytometry. Finally, the ability of platelets to respond to TRAP-6 stimulation in vitro was also determined (30).

\section{Patients and methods}

\section{Patients}

The group of 15 patients (14 men, 1 woman, mean age 59.3 yrs.) who uderwent PTCA for stenosis of myocardial arteries at the Department of Cardiosurgery, University Hospital, Charles University, Faculty of Medicine, Hradec Králové, was examined. The study was approved by Institutional Ethical Commitee and written informed consent was obtain from any participant. Patients were medicated $24 \mathrm{hrs}$. before PTCA by $100 \mathrm{mg}$ of acetylosalicylic acid in all but one exception who was medicated by low-molecular heparin (nadroparin) 11.4kI.U. subcutaneously. Anxiolytic drugs concomitantly with antihistaminic drugs were administered to all patients immediately before PTCA. Average dose of heparin during PTCA administered to each patients was 11060 I.U. After the procedure 10 patients were treated by $500 \mathrm{mg}$ of ticlopidine, heparin 3000 I.U. was intravenously administered to two patients and one patients was treated by $10.9 \mathrm{ml}$ nadroparine subcutaneously. Long-lasting medication of patients included acetylosalicylic acid (13 patients), nitrates ( 13 patients), $\beta$-blocators ( 13 patients), hypolipidemics ( 9 patients), ACE-inhibitors ( 6 patients), antiarythmiacs ( 3 patients) and many others. Minimal (maximal) number of long-lasting medication administered to one patients in patients group was 6 (13), mean 9 . 
For important characteristics of the patient's group see the Tab. 1.

As a control, peripheral blood samples obtained from a group of 25 adults ( 17 women, 8 men) recruited from the staff members were examined. All volunteers were otherwise healthy, without any medication 2 weeks before blood sampling. The values of their blood leukocytes, blood cell count and platelet parameters were falling into the physiological ranges. Parameters of the patients and control group are shown in the Tab. 2.

Tab. 1: Patients underwent PTCA - essential parameters.

\begin{tabular}{|l|c|}
\hline gender (M:F) & $\mathbf{1 4 : 1}$ \\
age (yrs) (mean) & $\mathbf{5 9 . 5}$ \\
\hline weight (kg) (mean) & $\mathbf{8 3 . 5}$ \\
\hline height (cm) (mean) & $\mathbf{1 7 3 . 6}$ \\
\hline number of occluded coronary arteries & $\mathbf{9}$ \\
in one patient: 1 coronary artery & $\mathbf{5}$ \\
2 coronary arteries & 1 \\
3 coronary arteries & $\mathbf{3 6}$ \\
\hline total number of balloon dilatations & $\mathbf{6}$ \\
\hline localization of dilated stenosis: ACD & 3 \\
RC & 3 \\
RD & $\mathbf{5}$ \\
RIA & $\mathbf{2}$ \\
RIM & $\mathbf{2}$ \\
RMS & $\mathbf{1}$ \\
\hline administered dose of heparin on RMS & $\mathbf{1 1} 060$ I.U. \\
in 1 PTCA (mean) &
\end{tabular}

Abbreviations:

ACD - arteria coronaria dextra

$\mathrm{F}$ - female

$\mathrm{M}$ - male

PTCA- percutaneous transluminal coronary angioplasty

$\mathrm{RC}$ - ramus circumflexus

RIA - ramus interventricularis anterior

RIM - ramus intermedius

RMS - ramus marginalis sinister

$\mathrm{RD}$ - ramus diagonalis

\section{Method}

Three blood samples were drawn from each patient to VACUETTE $^{\mathrm{R}}$ (Greiner Labortechnik, Austria) tubes treated with citrate sodium. The first sample (A) was collected from vena brachialis before PTCA. The second sample (B) was an arterial blood obtained from balloon catheter located into proximity of arterial stenosis immediately after visualisation of coronary arteries by roentgen-contrasting compound. The third sample (C) was obtained from the same arterial catheter $10 \mathrm{~min}$ after PTCA or $10 \mathrm{~min}$ after stent placement, respectively (28). The samples were processed within 2 hrs. after collection. Carefully selected whole blood method to avoid an artifitial platelets activation was used $(2,12)$.

Blood samples from fasting healthy control were drawn from cubital vein at 8 to 9 o'clock a.m. Needles LUER 22G (Greiner Labortechnik, Austria) were used. The first $2 \mathrm{ml}$ of blood were used to determine basal hematological parameters (platelets count (PTC), mean platelet volume (MPV), platelet hematocrite (PCT), width of platelet volume distribution (PDW). Next $2 \mathrm{ml}$ of blood were used both for flow cytometry analysis and TRAP- 6 induced platelet activation in vitro.

To identify membrane molecules of platelets the panel of monoclonal antibodies purchased from Immunotech ${ }^{\circledR}$ (Immunotech - a Beckman Coulter Company, Marseille, France) was established. This panel includes either FITC or PE conjugated monoclonal antibodies against CD9, CD31, CD36, CD41, CD42a, CD42b, CD45, CD62P, CD63 molecules. Negative isotypic controls of the same subclasses were included in every analysis.

Blood samples were diluted by Sörensen phosphate buffer solution (PBS, pH 7.4) to obtain sample concentration $10 \times 10^{9}$ platelets/1 (2) and incubated with optimal concentration of monoclonal antibody for $20 \mathrm{~min}$. The sample was subsequently diluted by PBS and immediately analysed by flow cytometry. The same sample staining procedure was used to analyse platelets surface molecules after TRAP-6 induced activation in vitro. Flow cytometer Coulter ${ }^{\circledR}$ Epics ${ }^{\circledR}$ XL (Coulter, Fullerton, USA) equiped with software version Epics XL Flow Cytometry Work Station - System II $^{\mathrm{TM}}$ ver. 3.0. was used through the study.

Tab. 2: Comparison of selected parameters between patients underwent PTCA and healthy controls.

\begin{tabular}{|l|c|c|c|c|c|}
\hline parameter & $\begin{array}{c}\text { healthy controls } \\
\text { mean / SD }\end{array}$ & $\begin{array}{c}\text { patients } \\
\text { mean / SD }\end{array}$ & $\begin{array}{c}\text { comparison } \\
\text { L / S / H }\end{array}$ & $\begin{array}{c}\text { statistical } \\
\text { significance (p) }\end{array}$ & $\begin{array}{c}\text { statistical } \\
\text { test }\end{array}$ \\
\hline age & $\mathbf{3 2 . 0 4} / 9.69$ & $\mathbf{5 9 . 3} / 8.32$ & $/ /$ H & $<0.001$ & t-test \\
\hline PTC & $\mathbf{1 9 9 . 4 6 / 4 4 . 6 5}$ & $\mathbf{2 2 1 . 2 1} / 50.66$ & $/ \mathbf{S} /$ & 0.18 & t-test \\
\hline MPV & $\mathbf{8 . 0 9} / 0.24$ & $\mathbf{8 . 4 9} / 0.76$ & $/ /$ H & $<0.001$ & Mann-Whitney \\
\hline PCT & $\mathbf{0 . 1 4} / 0.04$ & $\mathbf{0 . 1 9} / 0.04$ & $/ /$ H & $<0.05$ & Mann-Whitney \\
\hline PDW & $\mathbf{1 7 . 3 6} / 0.68$ & $\mathbf{1 5 . 8} / 1.18$ & L / / & $<0.001$ & Mann-Whitney \\
\hline
\end{tabular}

$\begin{array}{lll}\text { Abbreviations: } & \text { L / S / H - low / same / high } & \text { PDW - width of platelet volume distribution } \\ & \text { MPV - mean platelet volume (fl) } & \text { PTC - platelets count }\left(\times 10^{9} / 1\right) \\ & \text { PCT - platelet hematocrite } & \text { SD - standard deviation }\end{array}$


The platelets population was delineated by forward scatter (FS) and side scatter (SS) parameters in whole unlysed blood. Platelet gate is always contaminated by the small fraction of debris formed from erythrocytes or leukocytes membranes and by large immune complexes (3). Intensity of fluorescence was measured in $\log$ scale by single parameter histogram (30). The activation of platelets was assessed by double immunofluorescence analysis using two parameters (dot-plot) histograms. Double positive $\mathrm{CD} 41^{+} \mathrm{CD} 62 \mathrm{P}^{+}$or $\mathrm{CD} 41^{+} \mathrm{CD} 63^{+}$platelets were considered as activated $(4,20)$. Expression of CD45 molecule ploted to SS parameter was used to delineate erythrocytes, leukocytes, aggregates of leukocytes and platelets. PLAs were identified as $\mathrm{CD} 45^{+} \mathrm{CD} 41^{+}$elements (21). The treshold of 5000 platelets was set up for a single analysis. It means that between 60000 and 140000 blood elements were analysed $(15,17,21)$ in a single determination. The relative proportion of PLAs was determined according to Matzdorff et al. (24) together with the evaluation of free platelets, platelet homoaggregates and platelet microparticles.

\section{Statistical analysis}

Statistical evaluation was performed by statistical software NCSS 6.0.21 (vers. 1996). Paired t-test was used in the case of normal distribution of experimental data. The nonparametric Wilcoxon or Mann-Whitney tests were used if the normal distribution of the data was lacked. KruskalWallis test with subsequent Bonferroni test were used to compare samples A, B, C.

\section{Results}

The methods using whole blood, platelet rich plasma (PRP) obtained either by simple sedimentation or by careful centrifugation, respectively, were compared in the preliminary study (results are not shown). As the best approach, technique using sodium citrate treated unseparated, unfixed whole blood processed during $2 \mathrm{hrs}$. after sampling was selected.

Statistically significantly decreased expression of all constitutively expressed membrane molecules was found on platelets of patients with PTCA in comparison with healthy controls (see Tab. 3 ). In an agreement with others $(5,18,19$, 24) significant increase in the activation between patient samples obtained before, during and after PTCA, respectively, was proven only for both CD62P and CD63 molecules (Tab. 4, Fig. 1,2) and PLAs (CD $45^{+} \mathrm{CD} 41^{+}$elements) formation. Statistically significant differences in the expression of other activation markers were not reached (Fig. 3).

The expression of both CD62P and CD63 molecules was significantly lower on platelets of patients before PTCA in comparison with healthy controls (statistical significance $p<0.05$ and $p<0.01$ respectively). There is statistically significant increase in the expression of CD62P molecule on platelets after PTCA in comparison with samples obtained before PTCA procedure $(\mathrm{p}<0.05)$ (Fig. 1). The gradual statistically significant increase in the expression of CD63 molecule before, during and after PTCA $(\mathrm{p}<0.001)$ was found (Fig. 2).

Tab. 3: Comparison of platelet membrane epression of selected molecules between patients with PTCA and healthy controls (relative values).

\begin{tabular}{|l|c|c|c|c|c|}
\hline parameter & $\begin{array}{c}\text { healthy controls } \\
\text { mean / SD }\end{array}$ & $\begin{array}{c}\text { patients } \\
\text { mean / SD }\end{array}$ & $\begin{array}{c}\text { comparison } \\
\text { L / S / H }\end{array}$ & $\begin{array}{c}\text { statistical } \\
\text { significance (p) }\end{array}$ & $\begin{array}{c}\text { statistical } \\
\text { test }\end{array}$ \\
\hline CD9 & $\mathbf{9 4 . 9 8 \% / 2 . 9 5}$ & $\mathbf{8 7 . 9 8 \% / 3 . 9 9}$ & L / / & $<0.001$ & Mann-Whitney \\
\hline CD31 & $\mathbf{9 2 . 7 8 \% / 2 . 9 7}$ & $\mathbf{8 7 . 1 0 \% / 4 . 2 4}$ & L / / & $<0.001$ & Mann-Whitney \\
\hline CD36 & $\mathbf{9 0 . 9 8 \% / 5 . 2 5}$ & $\mathbf{8 7 . 3 7 \% / 4 . 4 4}$ & L / / & $<0.05$ & Mann-Whitney \\
\hline CD41 & $\mathbf{9 5 . 6 2 \% / 2 . 2 3}$ & $\mathbf{8 8 . 0 9 \% / ~ 5 . 2 7 ~}$ & L / / & $<0.001$ & Mann-Whitney \\
\hline CD42a & $\mathbf{9 4 . 9 8 \% / 2 . 6 2}$ & $\mathbf{8 8 . 5 4 \% / ~ 5 . 0 6 ~}$ & L / / & $<0.001$ & t-test \\
\hline CD42b & $\mathbf{9 4 . 1 3 \% / 2 . 5 8}$ & $\mathbf{8 8 . 3 1 \% / 4 . 6 5}$ & L / / & $<0.001$ & $\begin{array}{c}\text { Kolmogorov- } \\
\text { Smirnov }\end{array}$ \\
\hline
\end{tabular}

Abbreviations:

$\mathrm{L} / \mathrm{S} / \mathrm{H}$ - low / same / high

SD - standard deviation

Tab. 4: Comparison of parameters of activations between A,B,C samples of patients and healthy controls (relative values and standard deviation).

\begin{tabular}{|c|c|c|c|c|}
\hline $\begin{array}{c}\text { activation } \\
\text { marker }\end{array}$ & $\begin{array}{c}\text { sample A } \\
\text { (before PTCA) }\end{array}$ & $\begin{array}{c}\text { sample B } \\
\text { (during PTCA) }\end{array}$ & $\begin{array}{c}\text { sample C } \\
\text { (after PTCA) }\end{array}$ & $\begin{array}{c}\text { healthy } \\
\text { controls }\end{array}$ \\
\hline CD62P & $\mathbf{0 . 2 2} \%(0.21)$ & $\mathbf{0 . 3 9} \%(0.31)$ & $\mathbf{0 . 6 7} \%(0.67)$ & $\mathbf{0 . 3 4} \%(0.18)$ \\
\hline CD63 & $\mathbf{0 . 2 6 \%}(0.23)$ & $\mathbf{0 . 4 5 \% ( 0 . 3 5 )}$ & $\mathbf{0 . 8 5 \% ( 0 . 4 9 )}$ & $\mathbf{0 . 4 5 \% ( 0 . 2 0 )}$ \\
\hline PLAs $\left(\right.$ CD45 ${ }^{+}$CD41 $^{+}$) & $\mathbf{1 3 . 5 7 \% ( 6 . 7 6 )}$ & $\mathbf{1 8 . 3 9 \% ( 1 0 . 9 6 )}$ & $\mathbf{2 3 . 6 3 \% ( 1 3 . 1 8 )}$ & $\mathbf{8 . 1 6 \% ( 3 . 4 3 )}$ \\
\hline
\end{tabular}

Abbreviations:

PTCA: percutaneous transluminal coronary angioplasty Sample A: before PTCA
Sample C: after PTCA or after stent placement Sample B: during PTCA 


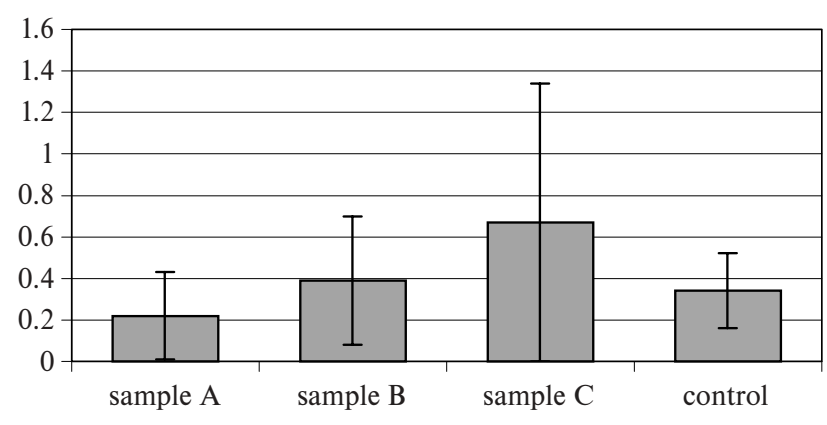

axis $\mathrm{x}$ - activation markers: CD41+CD62P+ elements in different groups

sample A: before PTCA

sample B: during PTCA

sample C: after PTCA

axis $\mathrm{y}$ - relative values of the expression in platelet population $(\%)$

Fig. 1: Comparison of CD62P expression on platelets between controls and three different patients samples obtained during the course of PTCA.

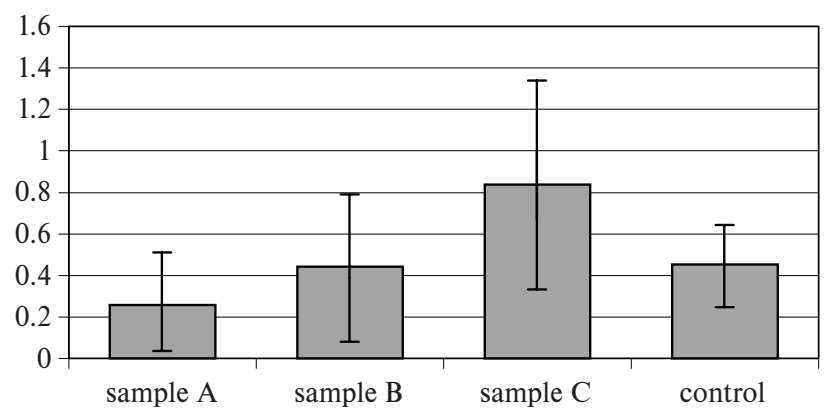

axis $x$ - activation markers $\mathrm{CD} 41^{+} \mathrm{CD} 63^{+}$elements in different groups sample A: before PTCA

sample B: during PTCA

sample C: after PTCA

axis $y$ - relative values of the expression in platelet population $(\%)$

Fig. 2: Comparison of CD63 expression on platelets between controls and three different patients samples obtained during the course of PTCA.

There were no statistically significant differences in the ability of patient's platelets to respond to TRAP-6 stimulation in vitro in comparison with controls. The only exceptions were significantly lower increase in the presence of PLAs $(\mathrm{p}<0.001)$, and platelet microparticles $(\mathrm{p}<0.01-0.05)$ both in whole blood and in platelets gate in blood samples collected before, during and after PTCA, respectively, in comparison with healthy controls after stimulation in vitro (Fig. 3).

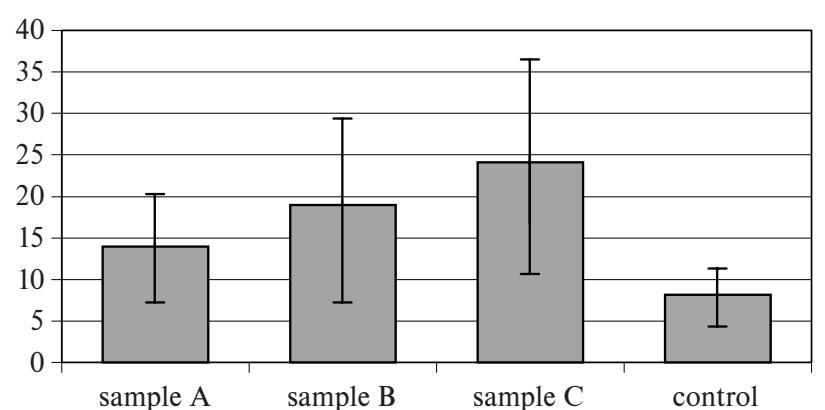

axis $\mathrm{x}$ - PLAs $\left(\mathrm{CD} 41^{+} \mathrm{CD} 45^{+}\right.$elements) in different groups sample A: before PTCA sample B: during PTCA sample C: after PTCA

axis $\mathrm{y}$ - relative values of PLAs (\%)

Fig. 3: Comparison of platelet-leukocyte aggregates (PLAs - CD $45^{+} \mathrm{CD} 41^{+}$elements) between controls and three different patients samples obtained during the course of PTCA.

\section{Discussion and conclusions}

The plasma or urine concentration of various humoral substances such as PF4, $\beta$-thromboglobuline or thromboxane $\mathrm{A}_{2}$ metabolites were previously used as markers of platelets activation in vivo (2). The commercial availability of monoclonal antibodies directed to platelet molecules together with a great progress in flow cytometry was the substantial breakthrough in immediate monitoring of platelets activation both in vivo and in vitro $(3,5,8,25)$. As the best approach, whole blood technique avoiding platelet separation and blood lysis, is commonly recognised. This method reflects the real situation in human body (2). As is stressed by Holada et al. (12), platelets rapidly response to changes in temperature, mechanical stress, exposure to artificial surfaces, and even to the traces of activation agents by rapid activation with consequent changes in the shape of platelets, aggregates formation, rearrangement of cytoskeleton and release of platelet granules. Careful choice of anticoagulant agents is prerequisited (3). For example EDTA does not interfere with phenotypisation of platelets but due to binding of $\mathrm{Ca}^{2+}$ ions EDTA interferes with platelets aggregation. Sodium citrate and heparin enable both the determination of platelets phenotypisation and platelets aggregation.

We found statistically significant lower expresion of all constitutively expressed surface molecules of patients undergoing PTCA in comparison with healthy controls. This findings could be a least partially explained by the medication of patients with PTCA. The average nine different drugs were administered to PTCA patients in contrast to no medication in controls. Among these drugs administration of antithrombotics (acetylosalicylic acid), nitrates, often in 
combination with $\beta$-blockers or calcium-channels blockers together with hypolipidemics was the apparent rule. Some of patients were also treated with low molecular heparin (nadroparin). In addition antithrombotics and anticoagulants were given to all patients before, during and after the course of PTCA. The influence of these drugs on platelets is discussed by Knight et al. (16), Koza et al.(19), Wahba et al.(24), Zhao et al. (33).

Surface membrane expression of activation molecules CD62P and CD63 were determinated by both single (17) and double immunofluorescence staining techniques $(4,20)$. The values obtained by those different methods did not reveal the significant differences in the case of CD62P expresion $(p=0.15)$, but were significantly different in the case of CD63 molecule. The expression of CD63 was significantly higher $(\mathrm{p}<0.05)$ using double immunofluorescence staining technique. In agreement with others we proved low expression of CD62P on resting and unstimulated platelets. Itoh and coworkers (14) and Furman and coworkers (7) refer that the expression of CD62P on platelets is $0.11 \pm 0.20 \%$ and $1.5 \pm 0.2 \%$ respectively in healthy donors. Expression of CD62P and CD63 in healthy controls $2.4 \pm 0.9 \%$ and $1.3 \pm-0.7 \%$, repectively was reported by Kolarov et al. (18). The expression of these markers in patients undergoing PTCA without complications was $2.5 \pm$ $1.0 \%$ and $1.1 \pm 0.9 \%$, respectively. The expression of CD62P and CD63 was futher increased in patients with PTCA suffered from complication (reocclusion, restenosis) to $11.3 \pm 7.3 \%$ and $2.6 \pm 2.4 \%$ respectively.

Matzdorff et al. (23) examined activation of platelets in vitro. The labeled MPs were used to assess the absolute number of platelets. The relative number of $\mathrm{CD} 62 \mathrm{P}^{+}$platelets was increased after in vitro activation but absolute number of $\mathrm{CD}_{62} \mathrm{P}^{+}$platelets was decreased after activation due to the overall fall in number of platelets. The similar increase in relative number and fall in absolute number was seen also for MPs. Platelet aggregates were increased in both relative and absolute numbers. Our results did not support these findings. According to Kolarov et al. (18), the decrease in the absolute number of platelets due to PTCA was significant - from $259 \pm 74.7 \times 10^{9} / 1$ to $238.2 \pm 57.9 \times 10^{9} / 1$.

$\mathrm{Li}$ and coworkers $(21,22)$ recommended the use of monoclonal antibody directed to GPIX (CD42a) or monoclonal antibody to GPIb $\alpha(\mathrm{CD} 42 b)$ for the reason that some epitopes on the GPIIb/GPIIIa (CD41/CD61) complex could be masked by platelets-leukocyte aggregates. Monoclonal antibody GPIX(CD42a) seems to be more resistant to proteolytic cleveage by elastases and cathepsin $G$ released from leukocytes which are activated after formation of PLAs. To target platelets MPs monoclonal antibody against CD41 was used through our study for the reason that all MPs are $\mathrm{CD} 41^{+} / \mathrm{CD}^{+} 1^{+}\left(\mathrm{GPIIb}^{+} / \mathrm{GPIIIa}^{+}\right)$, but only 50-60\% of MPs express CD42a (GP Ib), particularly large MPs (32).

Gawaz et al. (10) examined peripheral blood in patients with AMI (acute infarction of myocardium) who under- went PTCA before, and in the intervals 4, 8, 24, and 48 hrs. after this procedure. They concluded that there was transient decrease in the expression of active isoform of receptor for fibrinogen together with the expression of P-selectin $(p<0.01)$, decrease in the number of circulating platelets $(p<0.05)$ and increase in the number of MPs $(p<0.002)$.

The number of MPs in unstimulated blood is estimated to be in a range between 1-5\%, this number is equivalent to 2 500-14 000 MPs/ $\mu 1$ of unstimulated blood (24). The number of $\mathrm{CD} 45^{+} \mathrm{CD} 41^{+}$elements in $\mathrm{CD} 45^{+}$population is increased in our patients with PTCA in comparison with controls $(p<0.05)$, see Tab. 4, Fig. 3. There were no significant differences in the number of these elements before, during and after PTCA $(\mathrm{p}=0.86)$.

There are evidences that platelets are activated during PTCA $(5,11)$. We found significant increase in the expression of CD62P and CD63 molecules and concommitant increase in the number of PLAs. Inoue et al. (13) refered the significant increase in the activation of platelets and neutrophils in the patients with PTCA which was futher enhanced after stent placement. It is claimed that interaction between activated platelets and neutrophils could be responsible for the proliferation of the neointima leading ultimately to the restenosis after the placement of the stent. The activation of platelets associated with PTCA was also proven by DPPS (Duesseldorf PTCA Platelet Study) study (18).

The number of PLAs which is $14.7 \pm 11.4 \%$ in unfixed and unstimulated blood is increased to $19.9 \pm 13.3 \%$ after 3 hrs. of standing on bench (21). The same authors (21) conducted more detailed study to reveal the composition of PLAs. They found with the help of antiCD14 and antiCD16 monoclonal antibodies that $2.4 \pm 0.9 \%$ were heteroaggregates between platelets and lymphocytes, $7.2 \pm 1.6 \%$ heteroaggregates between platelets and monocytes and $3.7 \pm$ $0.3 \%$ heteroaggregates between platelets and neutrophils. They investigated circumstances which influence the formation and stability of PLAs. PLAs are more efficiently formed at $37{ }^{\circ} \mathrm{C}$ in comparison to laboratory temperature $22{ }^{\circ} \mathrm{C}$. Their formation is also increased in the presence of lysing solution and fixation solution. The same effect has also centrifugation and repeated washing of platelets. All above listed variables increase the formation of platelets 3-5 times.

Platelets are blood elements playing the pivotal role in the process of hemostasis. Platelets also contribute to the patophysiological mechanims. It is necessary to have standardised method to evaluate the activation of the platelets in vivo. This approach enables us to follow e.g. effects of the treatment strategy or to identify patients who are at the risk of restenosis after PTCA. In this sense it could be of a great clinical value to continue the long-lasting follow-up of platelets activation markers in patients after PTCA to delineate possible association with adverse effects such as a stent occlusion or restenosis.

The evaluation of the activation markers on platelets is highly specialized laboratory method. The appropriate in- 
terpretation of results relies on the close cooperation between clinician and cytometrist, because it is very likely that a lot of individual variables including patient medication significantly impair platelets. It is possible to prevent uncorrect interpretation by this collaboration $(2,3,25)$.

\section{Acknowledgement}

Autors are indebted to Josef Štásek, MD., PhD., for providing of blood samples from patients undergoing PTCA. The authors wish to thank to prof. Michael Aschermann, MD. DSc, F.E.S.C. for his critical reading of the manuscript.

\section{References}

1. Abrams $\mathrm{CH}$, Ellison N, Budzynski $\mathrm{AZ}$ et al. Direct detection of activated platelets and platelet - derived microparticles in humans. Blood 1990;75(1): 128-38

2. Abrams $\mathrm{CH}$, Shattil SJ. Immunological detection of activated platelets in clinical disorders. Thromb Haemostas 1991;65(5):467-73.

3. Ault KA, Mitchell J. Analysis of platelets by flow cytometry. Methods in Cell Biology 1994;42:275-94.

4. Ault KA, Rinder HM, Mitchell JG et al. Correlated measurement of platelet release and aggregation in whole blood. Cytometry 1989:10:448-55.

5. Cappani M, Prisco D, Antonucci E et al. The pre-procedural platelet state predicts clinical recurrence after coronary angioplasty. Int J Clin Lab Res 1999; 29(4):145-9.

6. Dehmer GJ, Nichols TC, Bode AP et al. Assessment of platelet activation by coronary sinus blood sampling during baloon angioplasty and directional coronary atherectomy. Am J Cardiol 1997;80(7):871-7.

7. Furman MI, Benoit MR, Barnard RM. Increased platelet reactivity and circulating monocyte-platelet aggregates in patient with stable coronary artery disease. J Am Coll Cardiol 1998;31(2):352-8.

8. Gasperetti CM, Gonias SL, Gimple LW et al. Platelet activation during coronary angioplasty in humans. Circulation 1993;88:2728-33.

9. Gawaz MP, Mujais SK, Schmidt B et al. Platelet leukocyte aggregation during hemodialysis. Kidney Int 1994:46:489-95.

10. Gawaz M, Neumann FJ, Ott I et al. Platelet function in acute myocardial infarction treated with direct angioplasty. Circulation 1996;93:229-37.

11. Gawaz M, Neumann FJ, Ott I et al. Role of activation-dependent platelet membrane glycoproteins in development of subacute occlusive coronary stent thrombosis. Coron Artery Dis 1997;8(3-4):121-8.

12. Holada K, Kružik P, Mikolajová M et al. The fluorescence flow cytometry technique in the diagnosis of hereditary platelet disorders-a case of Glanzmann's thrombasthenia, Cas Lek Cesk 1994;133(23):734-6.

13. Inoue T, Sohma R, Miyazaki T et al. Comparison of activation process of platelets and neutrophils after coronary stent implantation versus balloon angioplasty for stable angina pectoris. Am J Cardiol 2000;86(10):1057-62.

14. Itoh T, Nakai K, Ono $\mathrm{M}$ et al. Can the risk for acute cardiac events in acute coronary syndrome be indicated by platelet membrane activation marker P-selectin? Coron Artery Dis 1995,6(8):645-50.

15. Jakso P, Pajor L. Advantages of CD45 vs. side scatter based gating in the course of flow cytometric immuno-phenotyping in malignant hematologic diseases. Orv Hetil 1998;139(42):2509-13

16. Knight CHJ, Panesar M, Wilson DJ et al. Different effects of calcium antagonists, nitrates and $\beta$-blockers on platelet function. Possible importance for the treatment of unstable angina. Circulation 1997;95:125-32.
17. Koksch M. Durchflusszytometrische Diagnostik von Thrombozyten. Coulter Immunotech Diagnostics. Manual. Leipzig.1997:18-26.

18. Kolarov P, Tschoepe D, Nieuwenhuis HK et al. PTCA: periprocedural platelet activation. Part II of Duesseldorf PTCA platelet study (DPPS). Eur Heart J 1996; 17(8):1216-22.

19. Koza JM, Shankey TV, Walenga JM et al. Flow cytometric evaluation of platelet activation by ionic or nonionic contrast media and modulation by heparin and recombinant hirudin. Invest Radiol 1995;30(2):90-7.

20. Lefkowits J, Plow E, Topol EJ. Platelet glycoprotein IIb/IIIa receptors in cardiovascular medicine. N Engl J Med 1995;333(23):1553-9.

21. Li N, Goodall AH, Hjemdahl P. A sensitive flow cytometric assay for circulating platelet-leukocyte aggregates. Br J Haematol 1997;99(4):808-16.

22. Li N, Goodall A, Hjemdahl P. Efficient flow cytometric assay for platelet - leukocyte aggregates in whole blood using fluorescence signal triggering. Cytometry 1999;35:154-61.

23. Matzdorff AC, Berchner D, Kuhnel G et al. Relative and absolute changes of activated platelets, microparticles anded platelets, microparticles andation in vitro. Haemostasis 1999;28(6):277-88

24. Matzdorff AC, Kühnel G, Kemkes-Matthes B et al. Quantitative assessment of platelets, platelet microparticles, and platelet aggregates with flow cytometry J Clin Med 1998;131(6):507-17.

25. Michelson AD, Barnard MR, Krueger LA et al. Evaluation of platelet function by flow cytometry. Methods 2000; 21(3):259-70.

26. Michelson AD, Furman MI. Laboratory markers of platelet activation and their clinical significance. Curr Opin Haematol 1999,6(5):342-48.

27. Murakami T, Komiyama Y, Masuda M et al. Flow cytometric analysis of platelet activation markers CD62P and CD63 in patients with coronary artery disease. Eur J Clin Invest 1996;26: 996-1003.

28. Neumann FJ, Ott I, Gawaz M et al. Neutrophil and platelet activation at balooninjured coronary artery plaque in patients undergiong angioplasty. J Am Coll Cardiol 1996;27(4):819-24.

29. Scharf RE, Tomer A., Marzec UM et al. Activation of platelets in blood perfusing angioplasty-damaged coronary arteries. Flow cytometric detection. Arterioscler Thromb 1992;12:1475-81.

30. Šimák J, Holada K, Janota J et al. Surface expression of major membrane glycoproteins on resting and TRAP- activated neonatal platelets. Ped Res 1999;46(4):445-49.

31. Wahba A, Rothe G, Lodes $\mathrm{H}$ et al. Effects of extracorporeal circulation and heparin on the phenotype of platelet surface antigens folowing heart surgery. Thromb Res 2000;97:379-86.

32. Zdebska E, Wozniak, J, Dzieciatkowska A et al. In comparison to progenitor platelets, microparticles are deficient in GP Ib, GP Ib-derived carbohydrates, glycerophospholipids, glycosphingolipids, and ceramides. Acta Biochim Pol 1998; 45(2):417-28

33. Zhao L, Bath P, Heptinstall S. Effects of combining three different antiplatelet agents on platelets and leukocytes in whole blood in vitro. Br J Pharmaco 2001; 134(2):353-8.

Submitted May 2003.

Accepted August 2003.

PharmDr. Martina Loudová, Ph.D., Charles University in Prague,

Faculty of Medicine in Hradec Králové,

Department of Clinical Immunology and Allergology, Sokolská tr̆. 408, 50005 Hradec Králové,

Czech Republic. e-mail: mloudova@post.cz 